\title{
Nullius in Verba
}

\author{
R. Bergamaschi ${ }^{1}$ (1) F. Tonelli ${ }^{2}$
}

Received: 22 January 2022 / Accepted: 18 February 2022 / Published online: 8 March 2022

(C) Italian Society of Surgery (SIC) 2022

The Latin "Nullius in Verba" literarily meaning nothing in words was an invitation to believe no statements at face value. It was the motto of the Royal Society, which adopted it in London in the 1660s. Around that time, the Academia del Cimento implemented the motto "Provando e Riprovando" (Trying and Trying again) in Florence by accepting for publication only results that had been proven to be reproducible. Such mottos were an expression of these societies' attempts at withstanding Aristotelian syllogism and encouraging the unearthing of new knowledge. The latter effort spawned the unyielding demand for proof of scientific evidence, which has been haunting the surgical literature since. It is precisely the extent to which this may still be the case that prompts this editorial.

The first concern regards the interface between surgical innovation and patient safety. In 1979 the Belmont Report's conclusion read: "innovation and safety need not be antagonist" [1]. More recently, the IDEAL Collaboration was developed in an effort to report "first in human" through a process aimed at maximizing patient safety [2]. Despite this, a literature search using the term "first report surgery" yielded references in excess of 187 thousand averaging a dozen a year in the 1970s, but increasing to several thousand each year in the current decade [3]. Although a few of such claims may eventually prove to have validity, their defining characteristic seems more in line with a fishing competition than with any proof of their worth.

The next category for concern has been referred to as the bandwagon effect, which can be defined as the adoption of procedures that gained popularity notwithstanding the lack of robust evidence [4]. Bandwagons do grab the headlines due to a number of cognitive biases such as avoiding critical

R. Bergamaschi

rcmbergamaschi@gmail.com

1 Department of Surgery, New York Medical College, Valhalla, NY, USA

2 Department of Surgery, Careggi University Hospital, Florence, Italy individual thinking, wishing to conform to the majority, and rallying around a winner. Though, bandwagons in surgery do not generally appear out of thin air. There may be unresolved issues conducive to them. Nonetheless, the reader should be able to spot bandwagons on the basis of their patterns. For instance, offering a too easy one-size-fits-all solution to a complex clinical problem such as perforated diverticulitis with peritonitis. Another example can be the inability by other surgeons to reproduce comparable results such as it was the case of the Norwegian moratorium [5] on transanal total mesorectal excision. Moreover, the premise that doing justice to the literature has no longer meaning seems behind reinventing the wheel, for example, in rectal prolapse surgery. Closing the circle, editors and peer reviewers may play a role in railing bandwagons.

Most readers may have noticed in the last decade how authors often conclude their retrospectively designed manuscripts by stating that a randomized trial (RCT) should be performed to evaluate their results. This persistent trend begs the question as to whether authors simply make such statements to elude a conclusion or truly understand what randomization in surgery entails. In the latter case, there are a number of potential pitfalls deserving rigorous attention. Because the random design is biased in favor of the surgeon's pre-trial routine [6], surgeons' proficiency with both operations is a requirement to rule out learning curve biases. Clinical trials most commonly have a superiority or non-inferiority design with their results most commonly analyzed per protocol or as intent-to-treat [7]. In case the latter analysis is employed, the RCT should be critically appraised with regards to the impact of crossover (alias dictus: despite randomized to primary anastomosis actually undergoing Hartmann's procedure) on the clinical endpoint. An ad hoc meta-regression of RCTs could disclose how crossover with intent-to-treat analysis may significantly bias the results in favor of primary anastomosis. Although randomization is a relevant tool for minimizing confounders in surgical research, RCTs may not be necessary if the experiment is unwarranted, or inadequate [8]. 
An additional phenomenon affecting the current literature has been referred to as "spin" [9]. It merely consists of a series of methodological bias unintentionally leading to unwarranted interpretation of results with potential for patient harm. A concise insight into some systematic errors is provided herein. William of Ockham, a Franciscan who lived in medieval England was attributed the concept of Ockham's razor, a heuristic that favors simple rather than complex explanations. Hence, the metric of one outcome measure can retrospectively be chosen as a surrogate to justify an entirely new surgical procedure. In the 1920s, an electric plant located in Hawthorne at the outskirts of Chicago reported increased productivity following the decision to observe the employees at work. The impact of the workers' awareness of being observed became known as the Hawthorne effect, which can often be present in quality control studies. Jerzy Neyman, a statistician who helped establish the concept of hypothesis testing in research, became known for the practice of excluding severely ill patients resulting in an error of the estimated association between exposure and outcome. Voluntary registries are particularly at risk for Neyman's bias [10]. Robert McNamara, US Secretary of Defense during the Vietnam War became linked to the bias of making decisions on the basis of only one data set ignoring any other data points. In fact, excessive focus on event rates without adjusting for losses to follow-up may introduce the McNamara fallacy. Another bias initially called sunkcost fallacy became later referred to as Concorde fallacy, when funding the homonymous project was not discontinued even at the time when it became clear that there was no future for the aircraft. In research, the term can be applied when some authors are advocating for a surgical procedure despite overwhelming evidence against it.

At last, how not to mention the significance game permeating the current surgical literature! From the first application in medical sciences by Sir Ronald Fisher in 1935 [11], $p$ values have helped us measuring the probability that what we observe is not due to chance. It might not be the statistics that are at fault, but rather the authors who misuse them [12. It is possible that surgeons do not quite understand what the stats mean and (more so) do not mean. Hence, rather than getting rid of statistical significance, we should promote clinical significance by reporting the number needed to treat/ harm. Furthermore, one disconcerting reality is the sale of administrative databases containing de-identified patient data collected by clerks for billing purposes. Such data (not collected for research purposes and without a prospective hypothesis) are subsequently run into statistical software hoping for a "significant" $p$ value (p-hacking) to generate a post-hoc (retrospective) hypothesis. Regrettably, this is the reality of data-generated hypothesis (as opposed to hypothesis-generated data). Unlike administrative databases, the NSQIP (National Surgical Quality Improvement Program) data are accrued by research-trained nurses reporting to the American College of Surgeons, rather than to the authors of the intended manuscripts. The latter fact brings the added advantage of external validity of independent data reporting with the knowledge of standardized definitions.

In conclusion, the authors of this editorial wish to suggest surgeons to consider the following points: innovation in surgery should abide by safety first rules; independent individual thinking should be promoted to evaluate new procedures with a healthy dose of criticism in order to protect patients from bandwagons; in addition to surgery-specific aspects, RCTs require the uncertainty principle to be in place; the BIASIN (BIas Assessment in Surgical Innovation) questionnaire should be applied to research protocols to address potential methodological errors that could skew the study design; clinical (rather than statistical) significance should be considered to distance ourself from the significance game.

\section{Declarations}

Conflict of interest The authors declare that they have no conflict of interest.

Ethical approval This article does not contain any studies with human participants or animals performed by any of the authors.

Informed consent For this type of study formal consent is not required.

\section{References}

1. National Commission for the Protection of Human Subjects of Biomedical and Behavioral Research, Department of Health, Education and Welfare (DHEW) (30 September 1978). The Belmont Report. Washington, DC: United States Government Printing Office. Available at: https://videocast.nih.gov/pdf/ohrp_belmo nt_report.pdf [Accessed on October 11, 2021].

2. McCulloch P, Altman DG, Campbell WB et al (2009) No surgical innovation without evaluation: the IDEAL recommendations. Lancet 374:1105-1112

3. PubMed search. Available at: https://pubmed.ncbi.nlm.nih.gov/? term=first+report+surgery\&sort=date [Accessed on 10/19/2021]

4. Cohen L, Rothschild H (1979) The bandwagons of medicine. Perspect Biol Med 22(4):531-538

5. Wasmuth HH, Faerden AE, Myklebust TÅ et al (2020) Transanal total mesorectal excision for rectal cancer has been suspended in Norway. Br J Surg 107(1):121-130

6. van der Linden W (1980) Pitfalls in randomized surgical trials. Surgery 87:258-262

7. Iordache F, Bergamaschi R (2013) Randomized trials in colorectal surgery: a will o' the wisp. Colorectal Dis 15(8):923-925

8. Glasziou P, Chalmers I, Rawlins M, McCulloch P (2007) When are randomised trials unnecessary? Picking signal from noise. $\mathrm{Br}$ Med J 334:349-351

9. Boutron I, Dutton S, Ravaud P, Altman DG (2010) Reporting and interpretation of randomized controlled trials with 
statistically nonsignificant results for primary outcomes. JAMA 303:2058-2064

10. Hill G, Connelly J, Hebert R, Lindsay J, Millar W (2003) Neyman's bias re-visited. J Clin Epidemiol. 56(4):293-6

11. Fisher RA (1935) Statistical tests. Nature 136(3438):474

12. Wasserstein RL, Lazar NA (2016) The ASA's statement on $P$ values: context, process, and purpose. Am Stat 70(2):129-133
Publisher's Note Springer Nature remains neutral with regard to jurisdictional claims in published maps and institutional affiliations. 